\title{
Study Registration Date
}

National Cancer Institute

\section{Source}

National Cancer Institute. Study Registration Date. NCI Thesaurus. Code C93681.

The date (and time) the subject has been registered to the study. 